\title{
El Código de Ética, en el proceso de laudos arbitrales en el Perú
}

\author{
Gaidar Morales Alvarado, Renzo Zavala Llauce \\ HYM Consultores SAC, Perú \\ Essalud, Perú
}

\begin{abstract}
Resumen
El presente trabajo de investigación tiene como propósito contextualizar y analizar el Código de Ética del arbitraje desde su publicación a fin de establecer si existe un efecto en la resolución de laudos arbitrales. Se orienta bajo una metodología exploratoria descriptiva donde se observa el comportamiento de las variables entre el 2017 y el 2020. Los resultados obtenidos producto del análisis documental fueron, el Código de Ética como cuerpo normativo no tiene efecto sobre los procesos arbitrales, a tal razón, el Estado en el 2020 emite un Decreto de Urgencia para garantizar transparencia limitando a los árbitros en su accionar con reglas y criterios claros, sin embargo, hay mucho camino por recorrer y en ese proceso se debe fomentar, difundir y sancionar todo lo pautado en el Código de Ética.
\end{abstract}

Palabras clave: código de ética, laudos arbitrales, corrupción en el arbitraje, ética profesional.

\section{The Code of Ethics, in the arbitration awards process}

\begin{abstract}
The purpose of this research work is to contextualize and analyze the Code of Ethics of arbitration since its publication in order to establish if there is an effect on the resolution of arbitration awards. It is oriented under a descriptive exploratory
\end{abstract}


El Código de Ética, en el proceso de laudos arbitrales en el Perú

methodology where the behavior of the variables between 2017 and 2020 is observed. The results obtained as a result of the documentary analysis were, the Code of Ethics as a normative body has no effect on arbitration processes, for this reason The State in 2020 issues an Emergency Decree to guarantee transparency, limiting the arbitrators in their actions with clear rules and criteria, however, there is a long way to go and in this process everything established in the Code of ethics.

Keywords: code of ethics, arbitration awards, corruption in arbitration, professional ethics.

\title{
O Código de Ética, no processo de sentenças arbitrais
}

\section{Resumo}

\begin{abstract}
O objetivo deste trabalho de pesquisa é contextualizar e analisar o Código de Ética da Arbitragem desde sua publicação, a fim de estabelecer se há efeito na resolução de sentenças arbitrais. Se orienta bajo una metodología exploratoria descriptiva donde se observa el comportamiento de las variables entre el 2017 y el 2020. Los resultados obtenidos producto del análisis documental fueron, el Código de Ética como cuerpo normativo no tiene efecto sobre los procesos arbitrales, a tal razón, el Estado en el 2020 emite un Decreto de Urgencia para garantizar transparencia limitando a los árbitros en su accionar con reglas y criterios claros, sin embargo, hay mucho camino por recorrer y en ese proceso se debe fomentar, difundir y sancionar todo lo pautado en el Código de Ética.
\end{abstract}

Palavras-chave: código de ética, laudos arbitrais, corrupção na arbitragem, ética profissional.

\section{Introducción}

Actualmente, en el Perú la corrupción es percibida como uno de los principales problemas y está presente en el sistema de administración de justicia preferido en el ámbito privado, el arbitraje. El cual ha sido llevado al ámbito estatal sin mayores cambios.

De acuerdo con Guzmán-Barrón, Zúñiga y Seminario (2016), el arbitraje en las contrataciones públicas se fundamenta sobre la transparencia (consignado en Ley de contrataciones del Estado), toda vez que se realiza en vista del interés público. Es por esto por lo que se puede inferir que el arbitraje institucional es más transparente que el ad hoc, por las reglas establecidas y supervisadas en el desarrollo de sus 
actuaciones arbitrales. Sin embargo, consideramos que se han dado casos en los cuales la transparencia ha sido poco clara (ir punto 4).

Es así como la realidad permite que los riesgos de fraude y corrupción sean mayores en ambas esferas. Uno de los problemas es la designación repetitiva y constante de árbitros, que da como resultado laudos direccionados, y, sumado a ello, la transgresión de los principios y valores éticos por una mala praxis en un proceso arbitral (Barrón, 2017).

Según Anchante (2017), la preocupación de la comunidad profesional ha tenido como efecto la emisión de un código de ética a fin de limitar estos actos anti-éticos, sin embargo, resaltamos que el comportamiento moral de las personas -árbitros, partes, funcionarios, entre otros- va más allá de los códigos de ética.

Siguiendo con Anchante (2017), señala que el código de ética es aplicable a los arbitrajes que son sometidos al fuero arbitral del Centro de Análisis y Resolución de Conflictos PUCP, los procesos arbitrales ad hoc, así como, a los casos en que el Centro de Análisis y Resolución de Conflictos PUCP debe actuar como ente nominador de árbitros o autoridad decisoria de recusaciones. Lo dispuesto en el código es de aplicación obligatoria para los árbitros, integren o no la Nómina de Árbitros del CARCPUCP, incluyen los funcionarios y el personal del Centro, las partes, los asesores y sus abogados, en cuanto les sea aplicable.

Es importante precisar que, de acuerdo con el Decreto Legislativo № 1071, el arbitraje ejercido por una persona natural con plena capacidad de goce y ejercicio debe contar con pericia sobre aspectos legales y técnicos a fin de resolver una controversia. Vidal (2020) señala que este juez privado puede ser abogado o de otra profesión, con una retribución económica y sin ningún tipo de subordinación, y mantiene intacta la obligatoriedad de ejercer los principios de imparcialidad e independencia desde la aceptación del arbitraje hasta la emisión del laudo y actos procesales post laudo según también lo que señala el código de ética.

Las conductas muchas veces calificadas como "antiéticas" en el medio arbitral, dan cuenta precisamente de la incapacidad de la persona para razonar con autonomía y confrontar emociones morales con coherencia personal. Esto nos muestra lo imperioso de abordar los aspectos éticos más allá de establecer "Reglas de Ética", un documento que podría tener eco en la resolución de un laudo arbitral. Por tanto, nos preguntamos ¿el código de ética influye en la solución de controversias en un proceso arbitral?.

Es de precisar que el 29 de enero del 2020 se promulgó el Decreto de Urgencia $N^{\circ}$ 020-2020, mediante el diario oficial El Peruano, a razón de los casos de corrupción desde el arbitraje con el Estado, intentando resolver estos problemas por medio de regulaciones legislativas. La norma en mención modifica la Ley del Arbitraje a fin de establecer límites a la discrecionalidad de los árbitros y señalar conceptos claros sobre el conflicto de intereses. Según Purizaga (2020), el Estado pretende garantizar el proceso de arbitraje eliminando las prácticas corruptas con la definición de los criterios de publicidad y transparencia, toda vez que, el problema radica en la amplia 
discrecionalidad que tenían los árbitros para tomar decisiones y la falta de rendición de cuentas sobre el proceso. Cabe precisar que la norma señala que los arbitrajes ad hoc, se realizarán en circunstancias excepcionales.

Es importante señalar que mediante el Decreto de Urgencia $N^{\circ}$ 020-2020 y la Resolución Ministerial N 0159-2020, se aprobó la creación del Registro Nacional de Árbitros y de Centros de Arbitraje - RENACE a cargo del Ministerio de Justicia y Derechos Humanos.

En ese orden el Estado está realizando ajustes debido a la problemática que presenta el ejercicio profesional del árbitro y que este involucra su valores morales y éticos en su actuar. Es por esto, el objetivo del presente trabajo analizar si el código de ética ha tenido eco en el desarrollo del proceso arbitral.

\section{Aspectos generales de la ética}

\section{1. Ética}

La ética es una disciplina filosófica cuyo objeto de estudio es la moral. De acuerdo con la Real Academia Española (2020), señala que es parte de la rama de la filosofía que trata del bien y del fundamento de los valores. A modo de complemento, Cortina (2004) dice que la ética es un saber que pretende guiar la acción humana de manera racional en convivencia con la sociedad.

Para Millán y Vélez (2010) la ética se enfoca en el estudio de la moral, es decir, el análisis exhaustivo en comprender el comportamiento del individuo a través de teorías que argumentan las reacciones morales, de forma que se encuentran principios, reglas generales y normas que permiten el buen convivir de la sociedad.

En ese sentido, para Mancilla-Rendón, Díaz-Becerra, Morales-Alvarado (2018) el aspecto ético en las diferentes organizaciones implica factores relacionados al comportamiento de las personas y su entorno donde se desarrolla, acciones que deben estar comprendidas dentro de los valores morales y que deben estar dirigidas a la búsqueda del bien común. Sin embargo, la sociedad ha ido perdiendo la esencia de esos principios éticos y han sido vulnerados con actos negativos como la corrupción, y el abuso de autoridad o de confianza en perjuicio de la sociedad para el beneficio propio (Fondo Monetario Internacional, 2012).

\subsection{La moralidad}

Es preciso señalar de qué se trata la moral. La moral está orientada a la acción del sujeto, en relación con los otros, es decir, la responsabilidad de sus acciones frente a otros en libre desenvolvimiento en un grupo social (Santillana 2001 citado en Dasuky, 2010). 
Podemos señalar que la moral es un conjunto de creencias, costumbres, valores y normas de una persona o grupo social. En tanto que la moralidad es la operacionalización de la moral, es decir el conjunto de actos concretos que realiza una persona en una determinada sociedad.

\section{3. Ética profesional y el código de ética}

La teoría deontológica kantiana estudia el deber. Cuando esta teoría se aplica al estricto campo profesional hablamos de deontología profesional y es ella, en consecuencia, la que determina los deberes que son mínimamente exigibles a los profesionales en el desempeño de su actividad, plasmado mediante un código de ética. De esta forma, Yurén (2013) nos explica que la deontología profesional forma parte de un grupo cultural profesional, conformado por un conjunto de saberes, creencias, valores plasmados en la acción que orientan al ejercicio profesional.

Siguiendo a Yurén (2013), la expresión de la ética profesional se plasma en un código que va a regir la conducta o los valores, y que está plasmado en documentos formales cuyo propósito es explicar los principios, los valores y reglas de actuación de un ente o organismo.

La principal función de un código es servir de guía o advertencia para la conducta en situaciones específicas. Un código debe ser diseñado fundamentalmente para inspirar, dar coraje y apoyar a los profesionales éticos, pero también para servir de base para proceder contra los que actúan mal. En contraposición con los códigos legales, los deontológicos no deben sólo prohibir conductas, sino que deben tener un énfasis positivo, apostando por modelos deseables de conducta profesional. En este sentido, podemos distinguir en todos los códigos dos tipos fundamentales de normas: normas de prohibición y normas de orientación. Ambas deben conjugarse para formar un documento, que a la vez que marque claramente la línea de lo permitido y no permitido en el ejercicio de la profesión, señale a las más altas de las cimas de la excelencia profesional (Martínez, 2009).

\section{La regulación ética en los procesos de laudos arbitrales}

De acuerdo con el Reglamento de arbitraje vigente desde el 01 de enero del 2017 y aprobado por la Cámara de Comercio de Lima, en la sección "Reglas de ética" expone la ejecución de la normativa sobre el actuar del árbitro

En el Artículo 1 de las "Reglas de Ética" se señala que es de observancia obligatoria para los árbitros nombrados por las partes, terceros integren o no el registro de árbitros del "Centro", lo cual, es aplicable para todos los individuos involucrados en un proceso de arbitraje, así como, los abogados, asesores y representantes, por tanto, también son aplicables para los arbitrajes ad hoc (Cámara de Comercio de Lima, 2017, p. 84).

En cuanto al ejercicio profesional del árbitro, en los artículos 2, 3, 6, 7, 8, 9 y 10 se 
menciona que una vez aceptado el cargo no se debe mantener vinculo o interés de cualquier índole, así se evitan dudas sobre su independencia, se actúa con imparcialidad, y se sostiene una comunicación pulcra entre las partes y sus abogados, de tal forma que permita con celeridad una resolución justa y eficiente, durante el proceso arbitral, lo cual, significa que durante y después actuar con estricta confidencialidad y reserva sobre los hechos, por tanto, el árbitro debe preservar la integridad cumpliendo el debido proceso arbitral (Cámara de Comercio de Lima, 2017, pp. 85-86; Cámara de Comercio de Lima, 2017, pp. 88-91).

Siguiendo con las "Reglas", en el artículo se 4 señala que el árbitro debe cumplir con sus deberes generales como ejercer la imparcialidad e independencia, contar con las competencias de los casos en controversia, así como, contar con el tiempo y disponibilidad para llevar a cabo el arbitraje (Cámara de Comercio de Lima, 2017, p. 86).

En tanto que, el artículo 5 señala que toda persona que no cumple los artículos 2 y 3 debe revelar y abstenerse en aceptar el cargo (Cámara de Comercio de Lima, 2017, pp. 86-87). Durante del proceso arbitral debe comunicar sin dejar de omitir cualquier hecho o duda que pueda vulnerar la imparcialidad del proceso. Cabe precisar que, una vez aceptado el cargo, el árbitro no puede renunciar ni abandonar, salvo circunstancias imprevistas. Y, según el artículo 9, en caso se presente la renuncia, se debe asegurar la protección del interés de las partes en el arbitraje (Cámara de Comercio de Lima, 2017, pp. 90-91).

Por tanto, las reglas sobre la conducta y proceder antes, durante y después del arbitraje están claramente estipuladas en este documento, que es de precisar son de cumplimiento literal de cada artículo. Lo cual implica que el árbitro debe tener un sólido sistema ético-moral en su ejercicio profesional.

Cabe mencionar que el arbitraje en el Perú nació como una gran alternativa para la solución de controversias de forma rápida e imparcial, cumpliendo el debido proceso así como aminorando los costos que origina un largo proceso judicial, en síntesis un centro de arbitraje ofrece confidencialidad y seguridad en el desarrollo del proceso en un mínimo tiempo no obstante esta finalidad se ve transgredida cuando las personas cometen actos anti éticos con propósitos específicos transgrediendo la naturaleza de un centro de arbitraje.

\section{Metodología}

El presente trabajo se trata de un estudio de tipo exploratorio y descriptivo. Se analiza la literatura sobre ética, el código de ética en especial énfasis en el código de ética arbitral en el Perú vigente a partir del 2017.

Asimismo, se analizan los casos relacionados a laudos arbitrales desde la entrada en vigencia del código de ética arbitral. 


\section{Aplicación de casos en contra del código de ética}

\subsection{Investigan la actuación de 19 árbitros a favor de Odebrecht}

La Fiscalía Supraprovincial Corporativa Especializada en delitos de corrupción de funcionarios:

sospecha que los profesionales del Centro de Arbitraje de la Cámara de Comercio de Lima habrían cometido delitos de cohecho, lavado de activos y asociación ilícita. Habrían recibido "donativos" por sus decisiones. Un grupo de conocidos y reputados árbitros legales "habría aceptado y recibido donativo (dinero)" de Odebrecht para influir y emitir laudos arbitrales a favor de los consorcios o concesiones que integraba o dirigía la constructora brasileña. Esta es la sospecha por la cual el fiscal Marcial Paucar Chappa inició investigación a 19 árbitros del Centro de Arbitraje de la Cámara de Comercio de Lima, quienes intervinieron en 24 procesos arbitrales, favorables a la empresa brasileña. Los laudos arbitrales fueron sobre los proyectos $u$ obras de rehabilitación del Sistema de Agua Potable de Chimbote, IIRSA Norte, IIRSA Sur, tramo 2 y 3 y la carretera Callejón de Huaylas-Chacas-San Luis. La sospecha se sustenta en la declaración del colaborador eficaz 14-2017, conforme informó La República, el día de ayer. Este colaborador reveló a la Fiscalía que el árbitro Horacio Cánepa Torre negoció con el ingeniero Rony Loor Campoverde, directivo de Odebrecht, el pago del $1 \%$ del monto bruto de la controversia, para fallar a favor de la empresa, en 14 procesos arbitrales. Además, se le ofreció el pago de honorarios para él y los otros dos árbitros, por encima de los montos establecidos en los centros de arbitrajes y "donativos" para que los otros integrantes del Tribunal Arbitral también fallen a favor de la constructora brasileña. En total, según el colaborador eficaz, Cánepa recibió un pago total de 1.442.000 dólares, en una cuenta en la Banca Privada de Andorra, lo que supone que los laudos arbitrales resueltos a favor de Odebrecht habrían sumado unos 144.2 millones de dólares, sin IGV (La República, 2018).

\subsection{Caso Rodolfo Orellana}

Según Cruz (2019), para el diario El Comercio, el abogado Rodolfo Orellana presunto líder de una red articulada de criminales que han operado durante 12 años, se valió de los vacíos legales y sistematizó diferentes modalidades de operaciones criminales como la creación de liquidadoras que levantaban las hipotecas de los bancos, la emisión de las cartas-fianza de Coopex y finalmente el laudo arbitral.

En este último realizaba sus artilugios con el fin de expropiar a los privados de sus inmuebles, Paredes asegura que "Orellana se aprovecha de que existe un vacío legal en la Ley de arbitraje para crear un falso proceso arbitral que termina en un laudo declarado como cosa juzgada. El verdadero propietario recién se entera de esta operación cuando ya está inscrita en Registros Públicos y lo están por desalojar" (en Cruz, 2019), para tal efecto Orellana estaba respaldado por notarios, jueces, policías e incluso funcionarios municipales. 
En ese sentido, el laudo arbitral fue un instrumento para realizar una serie de actos ilícitos que usaba esta banda criminal para conseguir su propósito, claro dentro de todo este engranaje estaba inmerso el árbitro que no cumplió el deber ético profesional.

\section{Conclusiones y recomendaciones}

- Por lo tanto, el código de ética no influye en la resolución de laudos arbitrales. No basta generar normativas, toda vez que las acciones anti-éticas las cometen las personas en este caso el profesional competente. En ese sentido, se recomienda que la institución competente debe difundir, fomentar, invocar y sancionar al profesional competente.

- El arbitraje y la corrupción se pueden entrelazar de distintas formas: disputas derivadas de contratos obtenidos mediante corrupción, disputas derivadas de contratos para llevar a cabo prácticas corruptas, y disputas llevadas en un arbitraje corrupto (arbitros sobornados), en la medida que existan vacíos legales y profesionales con falta de valores éticos.

- El arbitraje corrupto no solo vulnera disposiciones jurídicas, sino que también en primera instancia transgrede las reglas éticas que están establecidas en el Reglamento aprobado por la Cámara de Comercio de Lima. Las preguntas que surgen a partir de ello son innumerables, lo que invita a la reflexión a los árbitros de seguir aplicando de manera diligente las normas. Ya que un laudo que ignora la corrupción es contrario al orden público nacional e internacional, por lo que las cortes nacionales terminará anulando y/o rehusandose a ejecutarlo y finalmente terminará perjudicando al arbitraje, porque "tienta"a las cortes nacionales a invadir los procedimientos arbitrales y conducir revisiones completas sobre el fondo.

\section{Referencias bibliográficas}

Anchante, M. (2017). Más allá de los códigos de ética en el arbitraje. Centro de Análisis y Resolución de Conflictos PUCP. Recuperado de http://carc.pucp.edu.pe/nota-deprensa/mas-alla-de-los-codigos-de-etica-en-el-arbitraje/

Cámara de Comercio de Lima. (2017). Reglas de ética. En Cámara de Comercio de Lima, Reglamento de arbitraje (pp. 83-92). Recuperado de https://apps.camaralima. org.pe/repositorioaps/0/0/par/reglamentoarbitraje2017/reglamento_ccl_2017.pdf

Cruz, R. (06 de junio de 2019). Rodolfo Orellana: ¿quién es y por qué el caso provoca tantas incautaciones de inmuebles?. El Comercio. Recuperado de https://elcomercio. pe/lima/judiciales/rodolfo-orellana-caso-incautaciones-inmuebles-noticia-ecpm642410-noticia/?ref=ecr 
Cortina, A. (2004). Ética sin moral. Madrid: Tecnos.

Dasuky, S. (2010). Cuatro versiones de la ética y la moral [notas de clase]. Universidad Pontificia Bolivariana, Medellín, Colombia. Recuperado de http://cmap.upb.edu.co/ rid=1GCFQ5KLN-T0G1 NL-9W/cuatro\%20versiones\%20de\%20la\%20\%C3\%a9tica. pdf

Decreto de Urgencia N 020-2020. Presidencia de la República del Perú (2020).

Decreto Legislativo. № 1071. Decreto Legislativo que norma el arbitraje. Organismo Supervisor de las Contrataciones del Estado-OSCE (2008). Recuperado de https:// portal.osce.gob.pe/arbitraje/sites/default/files/Documentos/Legislacion_aplicable/ DL-1071-ley-que-norma-el-arbitraje.pdf

Fondo Monetario Internacional (2012). Transparencia fiscal, rendición de cuentas y riesgo fiscal. Departamento de Finanzas Públicas y Departamento de Estadística. Recuperado de https://www.imf.org/external/spanish/np/pp/2012/080712s.pdf

Guzmán-Barrón, C., Zúñiga R., Seminario, C. (2016). Ética en el Arbitraje de Contrataciones Públicas: Problemas y Soluciones. Arbitraje PUCP, 6, 94-116. Recuperado de http://revistas.pucp.edu.pe/index.php/arbitrajepucp/article/ view/17029/17327

La República. (04 de mayo de 2018). Investigan la actuación de 19 árbitros a favor de Odebrecht. La República. Recuperado de https://larepublica.pe/politica/1237520investigan-actuacion-19-arbitros-favor-odebrecht

Martínez, F. (2009). La deontología: el fundamento de los colegios profesionales. Profesiones, 120, 14-15. Recuperado de: http://www.profesiones.org/var/plain/ storage/original/application/7f7177c469f7b6d1f729fbe712cf30bd.pdf

Mancilla-Rendón, E., Díaz-Becerra O. Morales-Alvarado, L. (2018). El Código de Ética profesional del Contador Público en Perú y México. ECONÓMICAS CUC, 39(2), 109128. Recuperado de https://doi.org/10.17981/econcuc.39.2.2018.07

Millán, A. Vélez, O. (2010). Ética y ciudadanía. Los límites de la convivencia. Lima: UPC.

Purizaga, L. (2020). Perú se blinda de los falsos arbitrajes con un decreto de urgencia. LexLatin. Recuperado de: https://lexlatin.com/reportajes/peru-blinda-falsosarbitrajes-decreto-urgencia

Resolución Ministerial № 0159-2020-JUS. Registro Nacional de Árbitros y de Centros de Arbitraje - RENACE, Ministerio de Justicia del Perú (2020).

Real Academia Española (2020), Diccionario de la lengua española, 23a ed. Recuperado de: https://dle.rae.es/\%C3\%A9tico

Vidal, R. (11 de mayo de 2020). La función arbitral en los arbitrajes de contratación pública. La Ley. Recuperado de: https://laley.pe/art/9690/la-funcion-arbitral-en-losarbitrajes-de-contratacion-publica

Yurén, T. (2013). Ética profesional y praxis: Una revisión desde el concepto de" agencia". Perfiles educativos, 35(142), 6-14. 
Fecha de recepción: 31/03/2021 Fecha de aceptación: 16/05/2021 Correspondencia: gaidahymconsultores@gmail.com, gestoresconsultoresperu@gmail.com 